\title{
OTIOTOMICS
}

Revista de economía, empresa y sociedad

Dossier: «Repensando la enseñanza de la economía en la universidad»

\section{La precaria enseñanza de la economía}

\section{Carlos Berzosa Alonso-Martínez}

Catedrático de Economía Aplicada de la Universidad Complutense de Madrid

RESUMEN La economía, tal como se enseña actualmente en la mayor parte de las universidades del mundo, es objeto de controversia. La formación económica que se recibe en las facultades y escuelas superiores es cuestionada desde determinados grupos de estudiantes y profesores. La crítica que se efectúa se ha intensificado con la crisis, pero ya se habían producido diversos brotes de protesta con anterioridad.

El sistema es cíclico, las crisis son inevitables y no son cosa del pasado, como el pensamiento dominante en economía había llegado a creer e infundir. La economía neoclásica, al desenvolverse con modelos de equilibrio, no es capaz de entender los procesos dinámicos de la economía, por lo que sus insuficiencias no se deben solamente a la incapacidad de predecir una crisis, sino también la de proporcionar las herramientas necesarias para la comprensión del funcionamiento de la economía.

La economía neoclásica no debe ser la única teoría actualmente en vigor, lo que no quiere decir que no se estudie el modelo IS/LM, pero hay que poner de manifiesto sus limitaciones, restricciones, además de que, como modelo de equilibrio, no responde a lo que sucede en la realidad, que es el desequilibrio y la vulnerabilidad. Por esto es necesario tener en cuenta otras aportaciones, como la marxista, keynesiana, schumpeteriana, institucionalista y poskeynesiana.

PALABRAS CLAVE economía neoclásica; historia económica; pensamiento económico; heterodoxia económica; economía poskeynesiana

\section{The precariuous teaching of economics}

SUMMARY Economics as it is currently taught in the majority of universities worldwide is the object of controversy. Various groups of students and lecturers are questioning the economics education being given by universities and colleges. The criticism being made has intensified in the wake of the crisis, but a number of different protests were also springing up earlier to this.

The system is cyclical and crises are inevitable, not a thing of the past as per the belief spread by the dominant school of thought in economics. Because of its inexstricable attachment to equilibrium models, neoclassical economics is incapable of understanding the dynamic processes in the economy, making it insufficient not only because of its inability to predict crises, but also because of its inability to provide the tools needed to understand the workings of the economy. 
Neoclassical economics should not be the only theory currently in force. This does not mean the IS-LM model should not be studied, but its limitations and restrictions must be highlighted; as an equilibrium model it does not correspond to what happens in reality, which is disequilibrium and vulnerability. It is thus necessary to take into account other ideas, such as Marxist, Keynesian, Schumpeterian, institutional and post-Keynesian theories.

KEYWORDS neoclassical economics; economic history; economic thinking; heterodox economics; post-Keynesian economics

\section{Introducción}

La economía, tal como se enseña actualmente en la mayor parte de las universidades del mundo, es objeto de controversia. La formación económica que se recibe en las facultades y escuelas superiores es cuestionada desde determinados grupos de estudiantes y profesores. La crítica que se efectúa se ha intensificado con la crisis, pero ya se habían producido diversos brotes de protesta con anterioridad.

La enseñanza de la economía ha ido variando a lo largo del tiempo, pero en las últimas décadas se observa una tendencia general para convertir la economía en un conocimiento que pretende asemejarse a las ciencias puras, como la matemática, y a las experimentales, como la física. Se da primacía, por tanto, a la enseñanza de instrumentos cuantitativos y a modelos abstractos sustentados en la teoría neoclásica. El rigor científico viene dado por la necesidad de cuantificación y formalización, dejando de lado todo lo que se considera, de un modo despectivo, literatura económica.

La insatisfacción que sienten los estudiantes ante esta situación es lógica, pues como han señalado en diferentes escritos y manifiestos, estos conocimientos se encuentran desligados de la realidad. Con este tipo de aprendizaje no se conoce el funcionamiento del sistema económico y apenas se sabe nada de la evolución y comportamiento de la economía mundial y de las diferentes regiones y países. Y lo que es más grave: con el instrumental teórico que se enseña no somos capaces de comprender el mundo real.

Cuestiones como la globalización, las desigualdades crecientes, la concentración de la riqueza y del poder económico, la existencia de la pobreza y del hambre, el ascenso de los países emergentes, los ciclos y crisis económicas, han desaparecido del objeto de estudio. De todos modos, cuando se abordan estas cuestiones se les dedica poco tiempo en las clases y escaso espacio en los manuales. Como consecuencia, el tratamiento suele ser muy pobre y bastante simplificador.

\section{La crisis y el fracaso de la economía}

Así las cosas, la crítica a la economía se intensificó tras la crisis iniciada en 2007 y que estalló en 2008, dado que la mayoría de los economistas fueron incapaces de predecirla, y ni tan siquiera pudieron advertir de los riesgos en los se estaba incurriendo y que podían desembocar en lo que realmente ha sucedido. Una exigua minoría, no obstante, sí fue capaz de predecirla y advirtieron de los riesgos que existían si se seguía en esa línea, debido a la fragilidad del sistema financiero, el tipo de crecimiento imperante, marcado sobre todo por el auge de la burbuja inmobiliaria, lo que acentuaba la vulnerabilidad como consecuencia del comportamiento y globalización de las finanzas (especulación, endeudamiento privado creciente, desregulación y exceso de emisión de titulaciones de alto riesgo), y la creciente desigualdad. Pero no fue el caso de la mayoría de la profesión académica, los ejecutivos de empresas, los servicios de estudio y los organismos económicos internacionales. La voz de esa minoría no fue escuchada.

La falta de preparación de los economistas a la hora de enfrentarse a acontecimientos no previstos y la incapacidad demostrada para entrever los riesgos que se avecinaban es en gran parte resultado de la formación 
que se recibe en el grado y el posgrado. Los economistas que ocupan cargos de responsabilidad en la toma de decisiones son producto de unos estudios insuficientes e incompletos para afrontar la dinámica social. La enseñanza de la economía está seriamente mutilada y debilitada por la hegemonía del modelo neoclásico, cuya manifestación más clara ha sido el estallido de la Gran Recesión.

La economía se ha convertido en una rama de las matemáticas, que se utiliza, junto con otros medios cuantitativos, como un fin y no como un medio para la comprensión de la realidad. Ha ido perdiendo su naturaleza de ciencia social. No se trata ni mucho menos, por mi parte, de cuestionar la importancia de las matemáticas, la estadística y la econometría en la formación que deben recibir los economistas. Pero un medio no se debe convertir en un fin, como tampoco hay que eliminar otros conocimientos que son imprescindibles, pero que hoy en día ya no se consideran como tales.

Chang (2015), por ejemplo, pone de manifiesto la desaparición de enseñanzas que deben ser claves cuando señala la progresiva desaparición en los planes de estudio de la historia económica. Otro tanto sucede con Sen (2014), que en el prólogo a una obra de Hirschman anota: «Describir este libro como una de las decisivas aportaciones de Hirschman es por tanto una declaración arriesgada. Y lo es más aún porque este es un libro -en realidad una breve monografía- sobre la historia del pensamiento económico, un asunto que recibe escasa atención hoy en día -y goza de un prestigio aún menor- y casi ha desaparecido de los programas de ciencias económicas en la mayoría de las universidades más importantes de todo el mundo».

La verdad es que se olvida el pasado tanto en la evolución de los hechos como de las ideas de los grandes economistas. Esto es una grave mutilación en la formación, así como en la cultura de los futuros economistas. Sin comprender el pasado difícilmente se puede entender el presente y sobre todo sacar provecho de las lecciones que la historia nos ofrece. Este desconocimiento conduce a cometer errores que ya se dieron en otros tiempos y no aprender de los economistas que nos precedieron es una grave deficiencia, pues es, en esencia, saber cómo afrontaron el conocimiento de la realidad de su tiempo y de los problemas que tenían ante sí.

Esto es tan básico como que resulta fundamental saber por qué se han dado los cambios de paradigma en el pensamiento económico dominante en cada época, de la misma manera que hay que estudiar las voces discordantes frente a la hegemonía de unas determinadas ideas. Y ello nos enseña, por un lado, que nunca ha habido un consenso entre los economistas a lo largo de la historia, ni tampoco en la actualidad, aunque siempre haya habido un paradigma predominante, y por otro, que la hegemonía de un pensamiento no es permanente, sino que ha sufrido cambios, lo que relativiza las teorías económicas.

La enseñanza actual no se preocupa del pasado, sino de enseñar unos modelos válidos para todo tiempo y lugar, como si la economía respondiera a leyes naturales que ha habido que ir descubriendo, modificando y mejorando. Y de ahí deducen que las leyes que dirigen la economía son permanentes, regidas por la eficiencia del mercado, cuando de hecho son producto de la acción de los hombres, que responden, a su vez, a coyunturas concretas y específicas, cambiantes, que modifican las relaciones económicas y sociales.

La renuncia a la historia, al conocimiento de la economía real, el abuso de las matemáticas, el uso de una teoría imperfecta y limitada es lo que se encuentra detrás de esta falta de previsión y de las medidas recomendadas durante la crisis, que tan desacertadas han resultado, sin que se haya propuesto un nuevo paradigma, tal como sucedió en las crisis de los años treinta y setenta del siglo pasado. Esto no quiere decir que todo cambio de paradigma sea positivo. Lo fue en los años treinta, pero no así en los setenta, pues mientras que las bases teóricas keynesianas y las intervenciones de los gobiernos proporcionaron en los países avanzados treinta años de prosperidad, las formulaciones monetaristas, expectativas racionales y de economía de oferta no han conseguido los índices de crecimiento anteriores, y han contribuido a crear un mundo más desigual, inestable e incierto.

\section{Las limitaciones de la economía convencional}

Conviene señalar que la economía actual no es el resultado de conocimientos acumulados a lo largo de la evolución histórica, sino que ha habido rupturas y, como ya se ha señalado, cambios de paradigma. Estos cambios a 
veces son profundos, como sucedió con la economía clásica y Marx, quien, aunque bebía de las fuentes clásicas, utilizó un aparato analítico diferente y llegó a conclusiones muy distintas acerca del funcionamiento del capitalismo. Posteriormente, la economía neoclásica ha supuesto un gran cambio con relación a la clásica, en cuanto a los conceptos y a los métodos: básicamente, se ha producido un abandono casi total del aparato analítico, pero se mantienen como hilo conductor los principios del liberalismo económico y las ventajas competitivas del mercado. Otra ruptura se produjo con Keynes en relación a la economía neoclásica, aunque la envergadura de esa brecha esté aún en disputa. Por último, cabe citar también la ruptura que tuvo lugar en los años setenta, cuando surgieron teorías antikeynesianas que ponían el énfasis en la eficiencia de los mercados, al tiempo que lo contraponían a los fallos del Estado.

Los grandes cambios en la teoría producidos durante el siglo xx han coincidido con las crisis de los años treinta y setenta. Esto contrasta con la situación actual, donde los economistas ortodoxos no han demostrado capacidad suficiente para modificar los supuestos básicos predominantes, pues lo que hoy se explica es la hegemonía de un modelo monolítico, sin dar cabida al conocimiento de los hechos reales actuales, ni a otras aportaciones económicas de interés, a la historia económica o al pensamiento económico. Estas insuficiencias son tan evidentes que se impone la necesidad de un cambio, pero, a pesar de la tozudez de los hechos, la ciencia económica convencional sigue imperturbable y no piensa ceder ante las grietas que la crisis ha provocado en la microeconomía y macroeconomía vigentes.

La justificación para seguir enseñando este modelo es que no ha sido este el que ha fallado provocando la explosión de la crisis, sino la ingeniería financiera, la avaricia, la codicia o el mal comportamiento de los dirigentes bancarios. Se trata, fundamentalmente, de dar interpretaciones de esta índole para salvaguardar la teoría tal como se enseña. $Y$ de paso, para no poner en cuestión el funcionamiento del sistema económico y de la fase actual caracterizada por la desregulación. Sin embargo, son demasiados fallos los que han tenido lugar para suponer que los errores o la falta de visión es lo que ha propiciado el desencadenamiento de los hechos que han conducido a la interrupción del ciclo expansivo.

La cultura de la satisfacción predomina y se sigue enseñando lo mismo como si la crisis no hubiera tenido lugar y no fuera con ellos. Es una muestra más del aislamiento en el que se encuentra sumida la economía como área de conocimiento y el alejamiento del comportamiento de la realidad. Se confía ciegamente en los mercados, como si estos no hubieran fallado, demostrando, una vez más, que están muy lejos de ser eficientes. E incluso en el supuesto ideal de que lo fueran, los modelos que generan no son precisamente socialmente deseables.

No obstante, la crisis ha generado una notable y abundante literatura que pretende explicar las causas que la han generado. Esto ha supuesto un interés por la historia, especialmente por la crisis de los años treinta y el debate que tuvo lugar entonces para encontrar remedios que condujeran a una salida. Esto ha propiciado una revitalización de los estudios sobre Keynes y en menor medida también sobre Kalecki, así como las controversias que tuvieron lugar, principalmente entre Keynes y Hayek.

Llama la atención también la recuperación que se ha hecho de Marx y de sus seguidores, debido principalmente a que este pensador fue un gran teórico sobre las crisis capitalistas, sin olvidar sus artículos de prensa, en los que analiza de una forma más coyuntural, pero muy aguda, las crisis que le tocaron vivir. Algunos de ellos parecen totalmente actuales.

Esta recuperación de la historia del pensamiento y del discurrir de los hechos se produce, sin embargo, entre una minoría dentro del mundo académico. No obstante, las contribuciones son sólidas y muchos de los artículos y libros tendrían que ser de obligada lectura en las facultades y escuelas de economía. Las corrientes de pensamiento -marxista, institucionalista, neokeynesianas, poskeynesianas y estructuralistas- adquieren un nuevo reconocimiento entre reducidos grupos de profesores y estudiantes, pero no en el mundo académico mayoritario, que sigue buscando la elegancia formal, aunque no sea explicativa de casi nada de lo que sucede en el mundo real.

En suma, para que la economía no quede encerrada en los despachos y aulas de los centros de enseñanza superior resulta necesario un cambio que en muchos casos supone volver a lo que constituyó el eje principal de las enseñanzas en nuestro pasado reciente. Los curriculum escolares están incompletos y muy mutilados al prescindir de la historia, del estudio de las instituciones y del análisis de los hechos reales. La formación del economista no se puede basar solamente en la formulación de modelos abstractos, sobre todo si la abstracción, 
aunque necesaria en toda teoría, no se hace teniendo en cuenta los factores más determinantes en la evolución y el funcionamiento económico.

Todo plan de estudios de grado que se precie debe contener matemáticas, estadística, econometría, teoría económica, economía internacional, economía mundial y del país en el que se estudia, organismos internacionales y sector público. En decir, una visión formativa global, dejando para los máster estudios más especializados y de economía avanzada. De este modo, los planes de estudio se enriquecerían frente a la pobreza actual. Y aunque resulta indudable que la interpretación del pasado y del presente admite enfoques muy diferentes -y para eso existe la libertad de cátedra-, lo que debe predominar siempre es el rigor académico.

\section{3. ¿Qué teoría económica enseñar?}

De lo dicho hasta aquí se deduce que para ser un buen economista no basta con conformarse con el estudio de instrumentos cuantitativos y de la teoría económica, sino que hay que complementarlo con otras disciplinas como las que he señalado. Ahora bien, ¿quiere esto decir que una enseñanza de estas características puede resolver el problema de la predicción de una crisis?

Esta no es una cuestión baladí, pues una de las críticas a la economía es su falta de capacidad predictiva frente a las crisis. Esto se debe, sin duda, a las limitaciones señaladas y al sesgo que ha tomado la enseñanza oficial en los últimos tiempos. No obstante, hay economistas relevantes, como Krugman, que consideran este fallo de predicción más como una consecuencia de una falta de visión que como una insuficiencia teórica. De hecho, Krugman ya venía anunciando la posibilidad de una crisis, frente a otros que no la vieron venir, y todos ellos utilizaban el mismo instrumental teórico, la síntesis neoclásica-keynesiana, lo que supone una defensa de la economía convencional frente a otros enfoques.

Otros analistas han puesto el acento en que un fallo de previsión no puede menoscabar la naturaleza científica de la economía. Se pone el ejemplo de los geólogos, que hasta ahora no han sido capaces de poder predecir cuándo va a ocurrir un terremoto y qué intensidad tendrá, y no por ello se cuestiona la geología como ciencia. Este argumento puede ser considerado como válido siempre y cuando a la economía actual no le diera por predecir. Las predicciones, por lo general, se desvían poco de lo que posteriormente sucede en tiempos de calma y de auge económico, aunque estos no sean muy abundantes, pero sus fallos quedan más en evidencia en periodos que anteceden a las crisis y en el propio desenvolvimiento de estas.

También hay quien señala, como Gerchunoff (2010), la imposibilidad de prever un fenómeno de esta naturaleza: «En los procesos sociales, los hechos se vuelven inevitables solo cuando han ocurrido; solo se sabe que un proceso de crecimiento y de bonanza económica es una burbuja cuando estalla; la noción de burbuja es ex post. Si una crisis no es una sorpresa para los contemporáneos, entonces no hay tal crisis».

Comparto solo parcialmente esta afirmación; mi disconformidad viene dada porque sí que ha habido autores que estudiaron la burbuja inmobiliaria antes de que estallara. Sus escritos están ahí, si bien es cierto que eso no supone que se previera el estallido tal como se ha dado -muchos hablaban de un aterrizaje suave-, ni tampoco la envergadura e intensidad de la crisis que ha tenido lugar.

Además, nunca sabremos qué es lo que realmente hubiera sucedido si se hubieran advertido los riesgos y, además, se hubieran tomado las medidas preventivas necesarias ante el peligro que se avecinaba, algo que se nos antoja realmente improbable por parte de los políticos responsables de tomar decisiones. En momentos de euforia, que suelen preceder al estallido de una crisis, ni los empresarios ni los políticos son conscientes de que los tiempos de bonanza se pueden acabar y tienden a minimizar el problema. En todo caso, los economistas podrían al menos haber salvado su reputación una vez que sus advertencias no fueran escuchadas. El problema es que la mayor parte de los economistas fueron incapaces de ver lo que estaba sucediendo. La minoría parecía predicar en el desierto.

Como dice el propio Gerchunoff: «En cualquier caso, espíritus ilustrados pueden abrigar esperanzas de que en medio del optimismo se esté incubando el huevo de la serpiente, pero estos espíritus no podrán impartir un consejo eficiente llamado a la prudencia a toda la sociedad, puesto que en tanto eficiente (y por tanto creíble), el 
consejo anula la crisis al instalar la frugalidad y la cautela allí donde reinaba el amor al riesgo. Es cierto que siempre hubo fallas de política y fallas regulatorias, y que el examen de esas fallas se puede aprender para el futuro, pero las crisis son inherentes a un mundo capitalista que se sostiene sobre los animal spirits y a un mundo democrático que se sostiene sobre la esperanza del progreso social».

De todo ello se deduce que el sistema es cíclico, que las crisis son inevitables y que no son cosa del pasado, como el pensamiento dominante en economía había llegado a creer y difundir. La economía neoclásica, al desenvolverse con modelos de equilibrio, no es capaz de entender los procesos dinámicos de la economía, por lo que sus insuficiencias no se deben solamente a la incapacidad de predecir una crisis, sino a su incapacidad de proporcionar las herramientas necesarias para la comprensión del funcionamiento de la economía. No es solamente un problema de visión, como dice Krugman, sino de algo más profundo, pues lo que fallan son los instrumentos teóricos.

La economía neoclásica no debe ser la única teoría actualmente en vigor, lo que no quiere decir que el modelo IS/LM deje de estudiarse, pero hay que poner de manifiesto sus limitaciones y restricciones; como modelo de equilibrio no responde a lo que sucede en la realidad: el desequilibrio y la vulnerabilidad.

Por esto es necesario tener en cuenta otras aportaciones muy útiles, como pueden ser las del economista poskeynesiano Minsky, cuyas contribuciones al conocimiento de la fragilidad del sistema financiero son indispensables para entender mejor las causas de la crisis actual. Fallecido hace unos cuantos años, el reconocimiento a su obra le llegó después de muerto, pues la mayor parte de los libros sobre la crisis lo mencionan. Un autor seguidor de su obra, Steve Keen, ha sido considerado, por la encuesta que hizo la Fundación Revere entre cinco mil economistas, como el número uno en predecir la crisis actual. Su libro La economía desenmascarada es una de las mejores críticas que he leído de la economía neoclásica, a la vez que analiza las causas de la Gran Recesión, así como las limitaciones de Krugman a la hora de entender la crisis. En esta obra donde se ponen más claramente de manifiesto las insuficiencias del modelo de la síntesis neoclásica-keynesiana para comprender globalmente la crisis al tener, entre otras cosas, una visión muy parcial, puesto que no considera en su esencia el funcionamiento del sistema financiero.

\section{Bibliografía}

CHANG, HA-JOON (2015). Economía para el 99\% de la población. Barcelona: Debate.

GERCHUNOFF, P. (2009). «Prólogo». En: Bleger, L. et al. Crisis global: Una mirada desde el Sur. Buenos Aires: Capital Intelectual.

KEEN, S. (2015). La economía desenmascarada. Madrid: Capitán Swing.

SEN, A. (2014). «Prólogo». En: Hirschman, A. Las pasiones y los intereses. Madrid: Capitán Swing. 


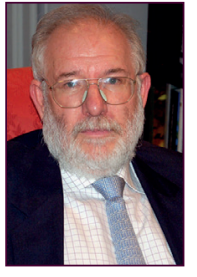

\section{Carlos Berzosa Alonso-Martínez}

berzosa@ccee.ucm.es

Catedrático de Economía Aplicada de la Universidad Complutense de Madrid

Catedrático de Economía Aplicada de la Universidad Complutense de Madrid y rector de dicha universidad del 2003 al 2011. Imparte enseñanza fundamentalmente en las disciplinas de Estructura Económica Mundial y Desarrollo Económico.

Es presidente de la Sociedad de Economía Mundial, que edita la Revista de Economía Mundial (REM), y director de la publicación Revista de Economía Crítica. Pertenece al consejo editorial de la revista mensual Temas para el Debate y ha dirigido la colección de economía de la editorial Síntesis.

Doctor Honoris Causa por la Universidad Nacional de Piura (Perú) y la Universidad de Tarapacá (Chile), ha sido presidente de la Fundación General de la Universidad Complutense y es académico de honor de la Real Academia de Doctores de España.

Los textos publicados en esta revista están -si no se indica lo contrario- bajo una licencia Reconocimiento-Sin obras derivadas 3.0 España de Creative Commons. Puede copiarlos, distribuirlos y comunicarlos públicamente siempre que cite su autor y la revista y la institución que los publica (autoría, nombre de la revista, institución editora); no haga con ellos obras derivadas. La licencia completa se puede consultar en http://creativecommons.org/licenses/by-nd/3.0/es/deed.es.

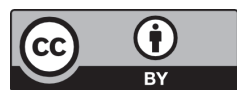

